\title{
A fiducial-aided data fusion method for the measurement of multiscale complex surfaces
}

\author{
Shixiang Wang, Chi Fai Cheung* and Mingyu Liu \\ Partner State Key Laboratory of Ultraprecision Machining Technology, Department of \\ Industrial and Systems Engineering, The Hong Kong Polytechnic University, Kowloon, \\ Hong Kong, China.
}

*Corresponding author: benny.cheung@polyu.edu.hk

Fax: (852)23625267

\begin{abstract}
Multiscale complex surfaces possessing high form accuracy and geometric complexity are widely used for various applications in fields such as telecommunications and biomedicines. Despite the development of multi-sensor technology, the stringent requirements of form accuracy and surface finish still present many challenges in their measurement and characterization. This paper presents a fiducial-aided data fusion method (FADFM), which attempts to address the challenge in modelling and fusion of the datasets from multi-scale complex surfaces. The FADFM firstly makes use of fiducials, such as standard spheres, as reference data to form a fiducial-aided computer-aided design (FA-CAD) of the multiscale complex surface so that the established intrinsic surface feature can be used to carry out the surface registration. A scatter-searching algorithm is employed to solve the nonlinear optimization problem, which attempts to find the global minimum of the transformation parameters in the transforming positions of the fiducials. Hence, a fused surface model is developed which takes into account both fitted surface residuals and fitted fiducial residuals based on Gaussian process modelling. The results of the simulation and measurement experiments show that the uncertainty of the proposed method was up to $3.97 \times 10^{-5} \mu \mathrm{m}$ based on a surface with zero form error. In addition, there is a $72.5 \%$ decrease of the measurement uncertainty as compared with each individual sensor value and there is an improvement of more than $36.1 \%$ as compared with the Gaussian process based data fusion technique in terms of rootmean-square (RMS) value. Moreover, the computation time of the fusion process is shortened by about $16.7 \%$. The proposed method achieves final measuring results with better metrological quality than that obtained from each individual dataset, and it possesses the capability of reducing the measurement uncertainty and computational cost.
\end{abstract}

Keywords: multiscale complex surface; precision surface measurement; data fusion; fiducial; multi-sensor

\section{Introduction}

Freeform optics, especially multi-scale freeform surfaces, are widely used in many fields such as advanced optics and biomedical engineering [1] due to their excellent 
performance in optical functionalities [2]. To ensure the performance of these advanced surfaces, advanced manufacturing technologies such as ultra-precision diamond cutting, milling and precision measurement technology are critical for the quality control of these surfaces. Many measurement instruments and principles, ranging from coordinate measuring machine (CMM) and interferometers to scanning electron microscopy, have been applied to fulfill different requirements and improve reliability or reduce uncertainty. However, with the increasing complexity of freeform surfaces, relatively few of these devices are capable of fulfilling all the measuring tasks because of the limitation of their own technology in terms of levels of resolution, measuring range and accuracy. For example, light scanners do not easily measure structured surfaces with sharp features, and touch probe-based coordinate measuring machines cannot capture microstructure information. Hence, multi-sensor data fusion using a common format to represent several datasets obtained from different sensors was developed to evaluate the metrological evaluation [3].

There are two main operations in the data fusion process, which are registration and data fusion. Registration is a process that unifies the dataset sources into a common coordinate system. To achieve this, an imaging sensor, a white light sensor and a tactile scanning sensor were integrated into a Werth VideoCheck UA 400 multisensor coordinate measurement machine[4]not only avoided misalignment but also possesses the ability to measure complex multi-scale surfaces. However, it is common for many measurements that the datasets are obtained from different coordinate measurement systems. Although the Iterative Closest Point (ICP) method [5] and its variants have been widely used for surface or discrete point matching/fitting [6], these methods are reported to be easily trapped in their local minimum and are very sensitive to the initial values of the given transferring parameters [7]. Some researchers have made use of intrinsic independent and immovable surface features, such as curvatures [8-11], to find corresponding pairs among data sources. These approaches have achieved fruitful results but they are still limited by their uncertainty and sensitivity to the machined structure [12].

The fusion process generates high quality unique information from all the datasets with different resolutions and different uncertainties in the overlapping area. Ye et al. [13] used the invariance of the curvature radius to obtain a good registration result and applied an optimal stitching planning method that is able to achieve a submicrometer level of stitching accuracy. The weighted fusion method [14, 15], which also sets the measured datasets in a linear system, has also been used in many studies, but the fusion results are obtained from minimizing the weighted summation of each system. Wang [16] analyzed four weighted fusion methods and their uncertainties were also investigated. The state-of-the-art-data fusion process focused on building models of the residuals of the datasets by using the Gaussian process [17, 18]. In this method, the residuals are defined by a linear measuring system so that the mean and covariance function of the Gaussian Process (GP) model can represent the fused results. However, these methods may be still used on simple surfaces, which are based on a linear system and are time-consuming. B-spline model is another developed technique to represent complex local geometry. Ren [19] employed B-spline to 
reconstruct a measured surface that had removed the residual. Results of the investigation indicate that this method has technological merit in terms of reducing computation cost and fusing complex surfaces. One drawback of the B-splines is that the unknown control points, or knots, require sophisticated optimal techniques.

This paper therefore presents a new method called fiducial-aided data fusion method (FADFM), which addresses the key problems in the multi-sensor data fusion, including improvement of the accuracy and robustness of the surface registration, reducing uncertainty and computational cost of the fusion process. Comparison experiments have been conducted for the existing methods based on both simulation study and experimental testes so as to verify the validity of the proposed method.

\section{Fiducial-aided data fusion method}

The proposed FADFM was purposely developed to improve the quality of the dataset from the following two aspects:

1) Enhance the surface registration step, which unifies different coordinate frames including that of the designed surface and those of the measuring datasets into one common coordinate frame.

This method firstly makes use of fiducials as reference data to generate the fiducial aided CAD surface, which is provided for a robust process of surface registration. Fiducials such as standard balls are designed and mounted on the fixture and they are able to provide intrinsic surface features.

2) Reduce the uncertainty and computational time in the fusion process.

The measurement results of the fiducials can provide uncertainty information of the measuring instruments in their measuring workspace. The uncertainty information was firstly used to determine the prior information in the Gaussian process modelling and it was used to determine the weight of each dataset in the weighted mean fusion step.

As shown in Fig. 1, the proposed FADFM is composed of three processes, which are surface registration, fusion, and the merging process. It starts with the generation of the fiducial-aided computer-aided design (FA-CAD) by using standard balls surrounding the surface with different heights. In the second step, the fiducials are measured in the different coordinate frames and are transformed so that the generated transformation spatial parameters are then used to transform the surfaces into a common coordinate system. Hence, the registration forms residuals of measured surfaces and the fiducials in different measuring processes are obtained. It is interesting to note that the accuracy of surface registration is further improved and a robust process is performed since the intrinsic surface feature patterns are registered. In the third step, the errors of fiducials serve as a reference to determine their own measuring uncertainty. The residual of surface registration in each measuring process is fused by a weighted mean fusion based on the Gaussian Process (GP). In the final step, merging the fused surface error with the FA-CAD generated the fused surface. The core fusion algorithms are discussed in detail in the following sections. 


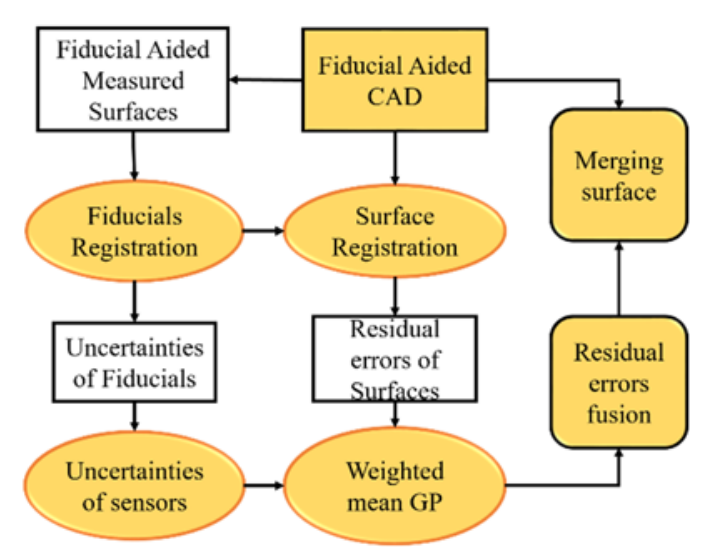

Fig. 1 Framework of the fiducial-aided data fusion

\subsection{Fiducial-aided surface registration}

Measured datasets from several uncorrelated sources are normally embedded in different coordinate systems. A surface registration process has to be carried out so that all the measured information is matched and unified in one common coordinate system. Matching is one of the most important and decisive processes in multi-sensor data fusion. It can be summarized as searching for an optimal vector $m$ with six spatial parameters so as to minimize by the least square function as shown in Eq. (1):

$$
\min \sum_{i=1}^{N}\left\|\mathbf{P}_{i}-\boldsymbol{T}(\boldsymbol{m}) \mathbf{P}_{i}^{k}\right\|^{2}
$$

where $\mathbf{P}_{i}$ are the points of the CAD; $\mathbf{P}_{i}^{k}$ are the cooresponding pairs in the $k$ th measured source, and $T$ is the coordinate transformation matrix determined by transformation parameter vector $m=\left(\alpha, \beta, \gamma, \mathrm{T}_{\mathrm{x}}, \mathrm{T}_{\mathrm{y}}, \mathrm{T}_{\mathrm{z}}\right)^{\prime}$, the first three parameters are the rotary angles and the others are translational offsets.

This nonlinear optimization problem (NLP) is able to be solved by using the Levenberg-Marquardt algorithm [20] or Gausss-Newton algrithm [21] by letting the Eq. (1) partial differential equal 0, i.e.:

$$
\sum_{i}^{N} 2\left(\mathbf{P}_{i}-\boldsymbol{T}(\boldsymbol{m}) \mathbf{P}_{i}^{k}\right) \frac{\partial\left(\mathbf{P}_{i}-\boldsymbol{T}(\boldsymbol{m}) \mathbf{P}_{i}^{k}\right)}{\partial \boldsymbol{m}}=0
$$

However, these methods are easily trapped into their local minima and the results are sensitive to the initial starting search for the six spatial parameters. To ensure the robustness and accuracy of the registration and feature-based surface descriptions, fiducials are used to carry out the surface matching [11]. Firstly, the fiducials in different datasets serve as intrinsic surface features to achieve a global minimizer $m$ by employing the OptQuest/NLP method which is a scatter searching method based on the multi-start framework [22] in Eq. (2). The description of the OptQuest/NLP method can be simply given by following the steps shown in Fig. 2 . 


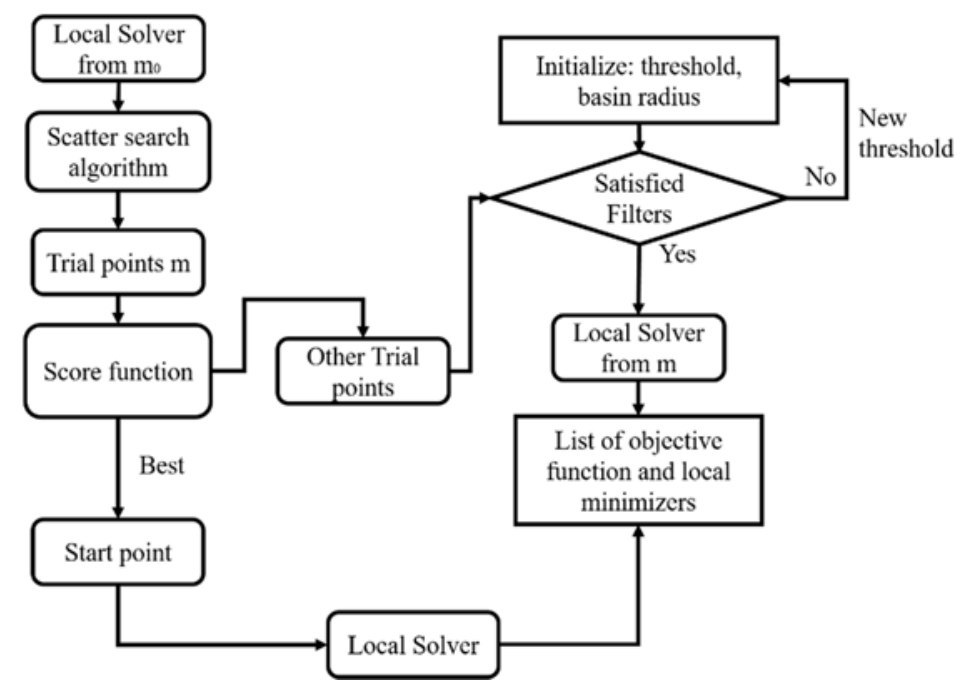

Fig. 2 Description of the Global Search Algorithm

It is clear that the OptQuest/NLP algorithm consists of two key stages. In the first stage, the given known point $m_{0}$ is used to run a NLP local solver so that an initial assessment of the radius of a basin of attraction can be recorded if the solver converges. Then the scatter search algorithm [23] is used to generate a group of trial points. These points are examined in a score function (penalty function). The bestscored points are prior to run the local solver and the results and themselves are stored in a linked list. The remaining trial points are checked in stage two with some initialized parameters such as basins and threshold referred to as the objective function values under valuable $m_{0}$ and the best start point. If the so-called trial points satisfy the distance and merit filter criteria, the solution list will be updated after a convergence of the local solver. Otherwise, the initialized threshold is increased in the loop. Finally, all the found solutions are sorted by an objective function from the lowest to the highest and the lowest solution is considered to be the global minimum $\boldsymbol{m}^{*}$ found by the fiducials. Hence, Eq. (1) can be solved robustly and the surface registration can achieve better results.

\subsection{Fiducial-aided weighted mean fusion based on the Gaussian Process}

It is assumed that the residual results obtained from the last fiducial-aided surface registration are distributed as Eq. (3):

$$
R_{k}(x, y)=f(x, y)+N\left(0, \sigma_{k} I\right)
$$

where $R_{k}(x, y)$ is a d-vector of the $k$ th residual results at points $(x, y) \in \mathrm{R}^{d}$ and $f(x, y)$ is the 'true value' that is being pursued in which the white noise is removed so that the normal distribution has 0 mean and $\sigma_{k}$ standard deviation. $\boldsymbol{I}$ is the 
identity matrix. The Gaussian Process (GP) [24] is employed to estimate the unbiased $\hat{f}_{k}(x, y)=G P\left(R_{k}\right)$. According to Wilson [24], the prediction values can be given the distribution over functions $\hat{f}_{k} \mid R_{k}$,

$$
\hat{f}_{k} \mid R_{k} \sim G P\left(V_{*}\left[V+\sigma_{k} I\right]^{-1} R_{k}, V_{* *}-V_{*}\left[V+\sigma_{k} I\right]^{-1} V_{*}\right)
$$

where $\hat{f}_{k}$ is the output values from the GP model predicted position, $V$ and $V_{* *}$ are the variance-covariance matrix at input and the predicted positions, respectively. $V_{*}$ is the covariance between the input and the predicted positions.

In the GP modeling, the zero mean function is used and the spectral Mixture kernel as described by Eq. (5) is chosen to model residual surface reconstruction:

$$
V\left(x, x^{\prime}\right)=\sum_{q=1}^{Q} w_{q} \cos \left(2 \pi \mu_{q}^{T}\left(x-x^{\prime}\right) \prod_{p=1}^{P} \exp \left\{-2 \pi^{2}\left(x-x^{\prime}\right)_{p}^{2} v_{q}^{(p)}\right\}\right.
$$

where $x, x^{\prime}$ are the inputs and evaluated positions with $\mathrm{P}=2$ dimensions, $\mathrm{Q}=10$ is the total numbers of mixture kernels, and $\mu_{q}$ and $v_{q}^{(p)}$ are the mean P-vector and covariance P dimensional diagonal matrix of the $\mathrm{q}$ th kernel.

Firstly, the fiducials (standard balls) in different measurement sources are evaluated and compared with the calibrated results with relatively higher accuracy so that the measurement uncertainty $\sigma_{k}^{\prime}$ in the whole measuring space can be achieved by Eq. (6):

$$
\sigma_{k}^{\prime}=\sqrt{\frac{\sum_{n=1}^{N} \sigma_{n, k}^{2}}{N}}
$$

where $N$ is the number of fiducials and $\sigma_{n, k}^{2}$ denotes the variance of the $\mathrm{n}$ th ball in the $\mathrm{k}$ th datasets. These obtained uncertainties can serve as Gaussian prior so that the hyperparameters $\sigma_{k}=\sigma_{k}^{\prime}$ in Eq. (4) are fixed.

Secondly, other kernel hyperparameters can be trained by minimizing the negative log marginal likelihood function by using BFGS/nonlinear conjugate gradients in the MATLAB toolbox [25].

Finally, a total of $\mathrm{K}$ datasets with different resolutions and sizes are given; the fused surface residuals can be derived as:

$$
\sum_{k=1}^{K} w_{k} \hat{f}_{k}, \quad \sum_{k=1}^{K} w_{k}=1
$$


where $w_{k}$ is the designed weight of each dataset source.

For the simple linear system expressed in Eq. (7), the uncertainty variance of the fused result can be denoted as:

$$
\sum_{k=1}^{K} w_{k}^{2} \sigma_{k}^{2}, \quad \sum_{k=1}^{K} w_{k}=1
$$

In order to obtain the minimum uncertainty, using the constrained nonlinear program conditions to solve the minimum solution of Eq. (8), the calculated weight can be set as:

$$
w_{k}=\frac{1 / \sigma_{k}^{2}}{\sum_{k=1}^{k} 1 / \sigma_{k}^{2}}
$$

\section{Experimental Investigation}

\subsection{Simulation and discussion}

The two main steps of the proposed method are fiducial-aided surface registration and fiducial-aided residual error fusion. A case study on two complex surfaces was designed and embedded in the same fiducial-aided structure. One optical freeform surface was the sinusoidal surface as defined by:

$$
\begin{aligned}
& z=0.2(\sin (2 x)+\cos (2 y))+15 \\
& x \in[-8,8], y \in[-5,5]
\end{aligned}
$$

Another surface was a more complex sinusoidal surface superimposed with microlens arrays as given by:

$$
\begin{aligned}
& z=15+0.05(\sin (0.5 x)+\cos (0.5 y))-\sqrt{r^{2}-\left(x-m_{p}\right)^{2}-\left(y-n_{q}\right)^{2}}-\Lambda \\
& x \in[-8,8], y \in[-5,5]
\end{aligned}
$$

where $\left(m_{p}, n_{q}\right)$ are the locations of the micro-lens which distribute in the grid $x \in[-6,6], y \in[-4,4]$ with $p=7$ and $q=5 ; r=8.5 \mathrm{~mm}$ and $\Lambda=8.48 \mathrm{~mm}$ are the lens radius and offset respectively.

There were six fiducials (standard balls with a diameter of $10 \mathrm{~mm}$ ) that were designed surrounding the designed surface. The coordinates of the centers were $(-6$, $10,14.5),(0,10,15),(10,0,15.5),(6,-10,14.5),(0,-10,15.5),(-6,-10,15)$ (unit is $\mathrm{mm})$ in the coordinate frame of the designed surface. Fig. 3 shows the two designed surfaces and the embedded fiducials. 
(a)

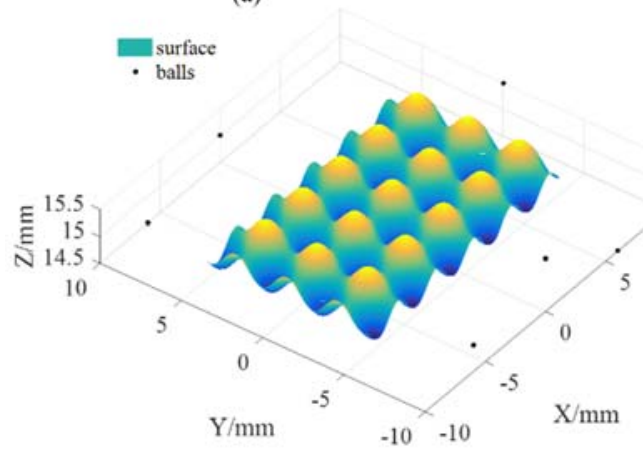

(b)

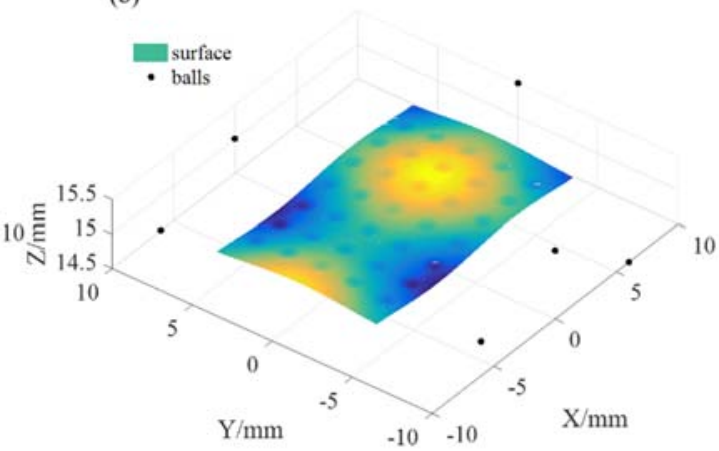

Fig. 3 Designed fiducial-aided (a) sinusoidal surface (b) micro-lens

In this case, two different sensors were used to measure the surfaces. Measuring noise with standard deviations of $1 \mu \mathrm{m}$ and $5 \mu \mathrm{m}$ were added respectively to the designed surface and fiducials so as to generate the measured datasets. Furthermore, the machined errors of the surfaces were set to zero. The measured points cloud of the surface denoted S1 was obtained with high-accuracy datasets and uniformly sampled with a spacing of $0.5 \mathrm{~mm}$, and S2 was sampled with a spacing of $0.2 \mathrm{~mm}$ in the lowaccuracy datasets. Then S1 and S2 were transformed with two different arbitrary known vectors $m=\left(\alpha, \beta, \gamma, \mathrm{T}_{\mathrm{x}}, \mathrm{T}_{\mathrm{y}}, \mathrm{T}_{\mathrm{z}}\right)^{\prime}$ as mentioned in Eq. (1) so that S1 and S2 were located in different coordinate frames with different uncertainties under different resolutions.

Fig. 4 shows the process of fiducial-aided surface registration by taking the microlens as an example. Both S1 and S2 were firstly transformed into the coordinate frame of FA-CAD by using the fiducials. Two surface residuals were then achieved after the surface registration. The GP model was used to reconstruct the measured residuals with the known measured uncertainty according to the measured results of the fiducials. The points were then resampled on the fused weighted mean system. 

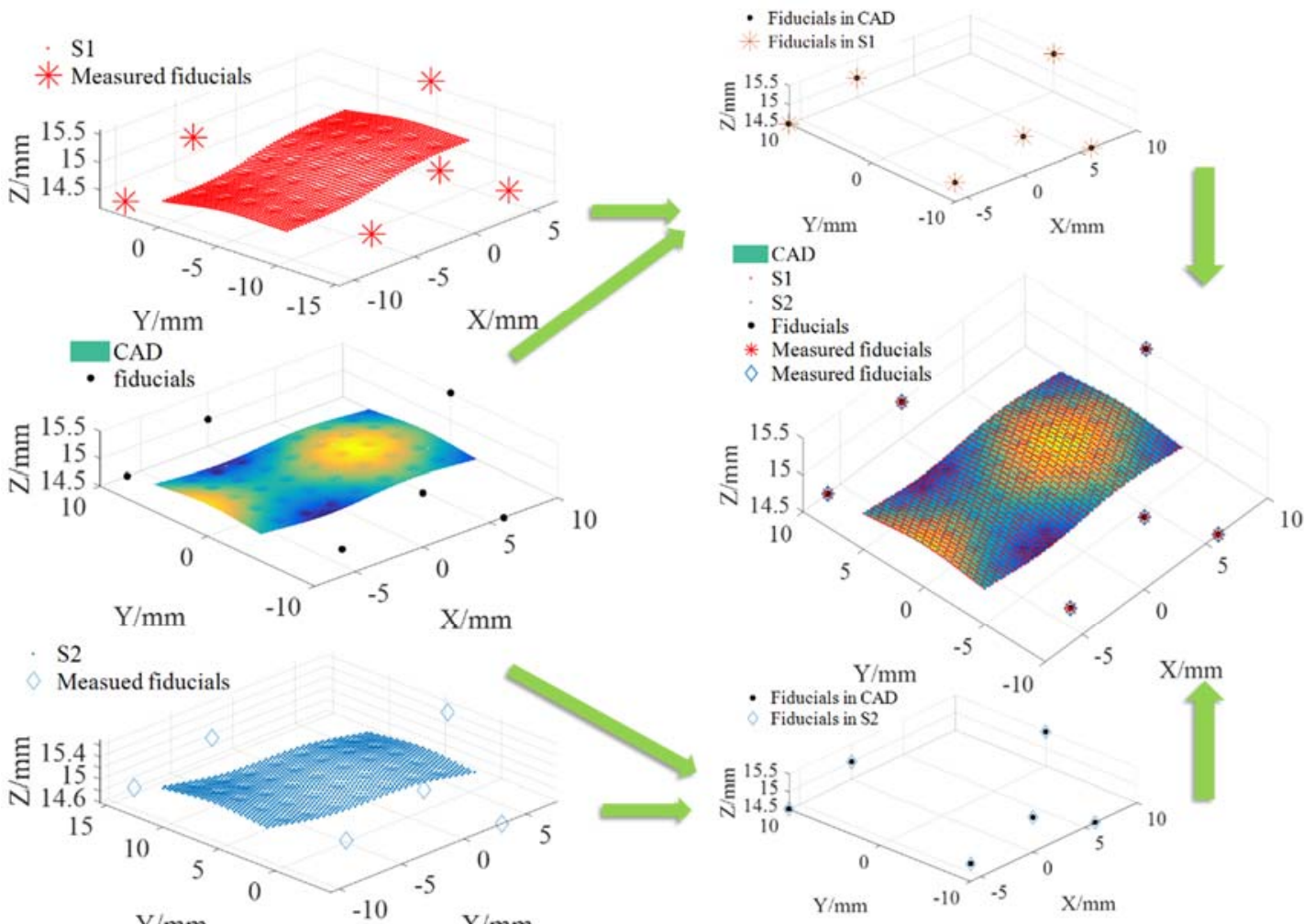

$\mathrm{Y} / \mathrm{mm}$

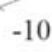

$\mathrm{X} / \mathrm{mm}$

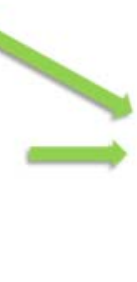

Fig. 4 Process of fiducial-aided surface registration

In order to evaluate the robustness and accuracy of the fiducial-aided surface registration, a total of 500 iterations of matching were repeated in the surface matching process using both the popular ICP and the proposed method. The results of the mean errors of the evaluated vector $m$ for its six spatial parameters are shown in Fig. 5 . It is clear that the proposed method performed better in terms of surface registration. This is due to the fact that the fiducials create intrinsic surface features which enhance the robustness of the registration process.

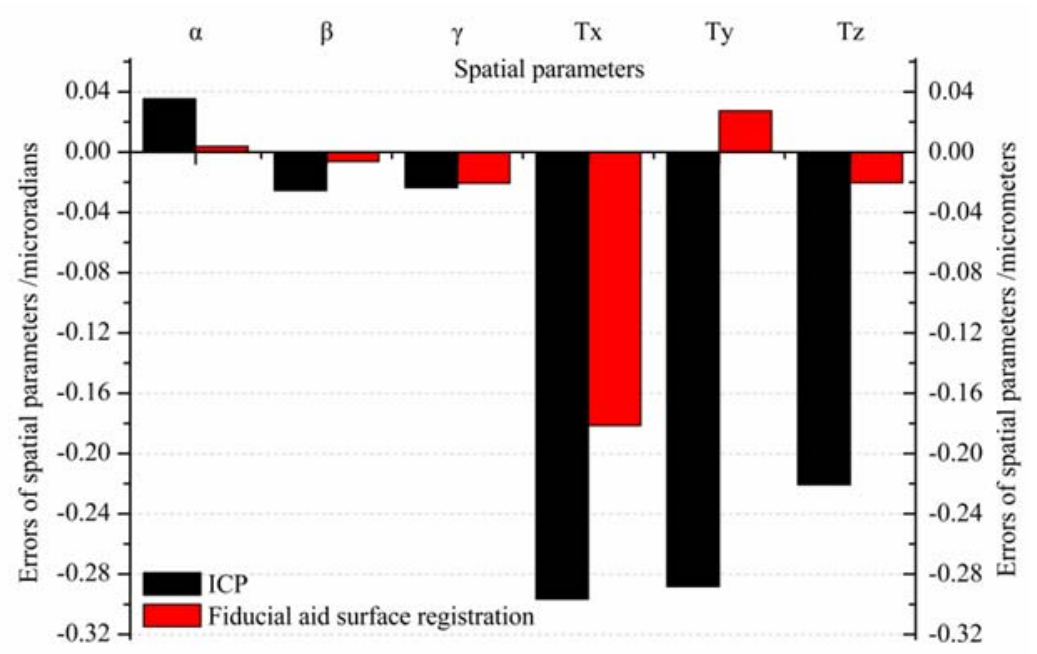

Fig. 5 Accuracy of the fiducial-aided surface registration compared with the ICP method in terms of six spatial parameters; $\alpha, \beta, \gamma$ are in micro-radians, and $\mathrm{T}_{\mathrm{x}}, \mathrm{T}_{\mathrm{y}}, \mathrm{T}_{\mathrm{z}}$ are in micrometers 
In the final stage, the performance of the FADFM was examined as compared with the result of the GP-based fusion method (GPBFM) which had no prior information of the uncertainty. It is noted that zero machined error was added to the surface, so the fused result should be close to zero. The simulation process was run using MATLAB software on an Intel Core i7 CPU 3.60 GHz PC with 16 GB of RAM.

There were 640 points on S1 and 4,000 points on S2. A total of 60,000 points (300 $\times 200$ in $x$ and $y$ directions) were sampled on the fused model. Fig. 6 shows the map of the fused form error. Table 1 shows the running time and fusion results by using the mean of root-mean - square (RMS) and peak-to-valley (PV) values after 50 repeated simulations. The results clearly show that the surface residuals sharply decreased over the original measurement results and the computation time was slightly shortened.

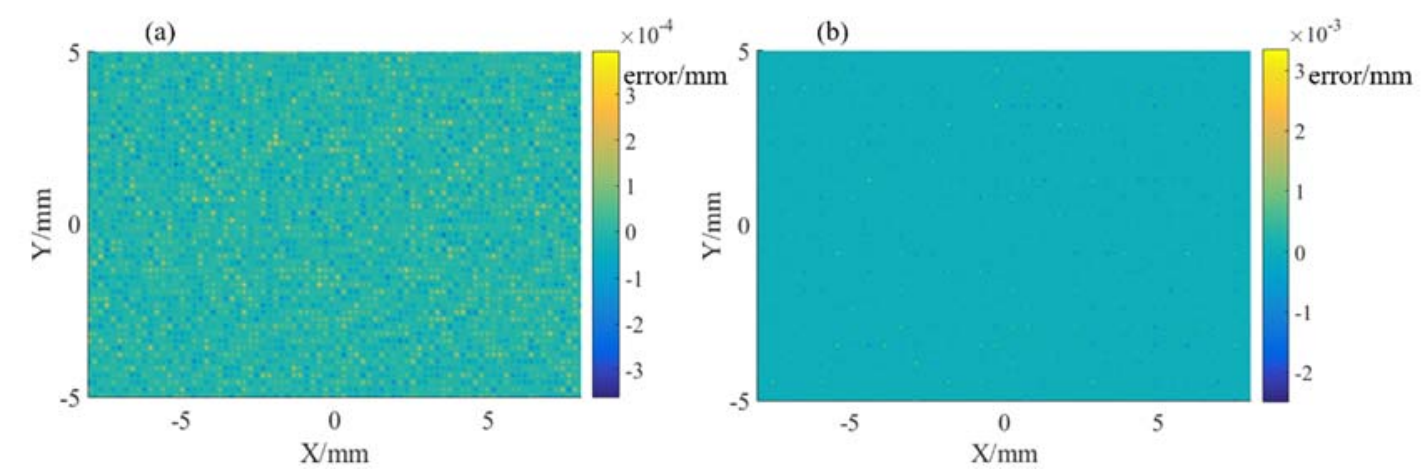

Fig. 6 Fused results of sinusoidal surfaces using two different fusion methods: (a) fiducialaided weighted mean fusion (b) GP-based fusion method

Table 1 Fusion results of the designed surface based on different fusion models after 50 simulations

\begin{tabular}{ccccc}
\hline & \multicolumn{2}{c}{ Sinusoidal surfaces } & \multicolumn{2}{c}{ Micro- lens } \\
\hline & FADFM & GPBFM & FADFM & GPBFM \\
\hline Mean of RMS $(\mu \mathrm{m})$ & $3.97 \mathrm{E}-5$ & $9.61 \mathrm{E}-02$ & $2.75 \mathrm{E}-05$ & $8.38 \mathrm{E}-02$ \\
Mean of PV $(\mu \mathrm{m})$ & 0.75 & 5.82 & 0.49 & 5.09 \\
Mean of Time $(\mathrm{s})$ & 29.2 & 34.5 & 35.4 & 39.8 \\
\hline
\end{tabular}

Notes: FADFM means fiducial aided data fusion method. GPBFM means GP-based fusion method.

\subsection{Discussion}

From the simulations shown in Fig. 6 and Table 1, the proposed method performed much better than the GPBFM. One reason is that the step of the surface registration is more robust with a lower uncertainty than the performance of the ICP method (see Fig. 5). Another reason is that enough information is used for the optimization in the core operations of the Gaussian process modelling.

In the FADFM, the RMS values in the two complex surfaces were reduced to 3.97 
$\mathrm{x} 10^{5} \mu \mathrm{m}$, and such low uncertainty indicates that the proposed FADFM almost found the 'accurate value' of the residual errors, which was zero in this case. On the other hand, the GPBFM also performed well although it only obtained $R M S$ values up to $9.61 \times 10^{2} \mu \mathrm{m}$ that were several thousand times larger than that for the FADFM. The computational time of the fusion process was shortened by about 16.7\% in FADFM, although both of the methods employed spectral mixture kernels which just require $O\left(P N^{P+1 / P}\right)$ operation [20]. This is due to the fact that the parameters to be optimized were decreased in the FADFM as compared with the GPBFM. It is clear from the simulated results that the proposed method has good capability of fusing datasets.

However, it is interested to note that the obtained peak - to - valude (PV) values in FADFM (up to $0.75 \mu \mathrm{m}$ ) was still relative large. This is due to the fact that one of the measurement datasets contained a lot of measuring noise, which caused the datasets to deviate from the true value due to bias. This is one of the limitations of data fusion that the fusion does not perform as well as expected when one of the datasets has large errors. The positive side is that the $P V$ values were at an acceptable level because of the balance of the weighted mean method and the uncertain information of the fiducials. Future work should focus on analyzing the effect of systematic errors in the datasets affecting the fusion results.

\subsection{Measurement experiment}

A fiducial-aided sinusoidal surface was machined to check the real surface fusion. The basic principle is that there are at least three non-collinear fiducials to provide the positions information. In order to reduce the systematic error, four standard balls were used as fiducials and the positions of each ball in FA-CAD are calibrated by a fiber sensor in the coordinate measuring machine (CMM) (Werth Videocheck from Gießen, Germany ) and the centres of the fiducials are listed in Table 2. Fig. 7(b) and Fig. 7(c) show details of the measured surface and the experimental setup.

Table 2 Positions of the centers of the fiducials in the designed model

\begin{tabular}{cccc}
\hline Sphere & $\boldsymbol{X}(\mathbf{m m})$ & $\boldsymbol{Y}(\mathbf{m m})$ & $\boldsymbol{Z}(\mathbf{m m})$ \\
\hline 1 & -4.71498 & -84.30512 & 8.97918 \\
2 & 83.70395 & -5.64727 & 4.08088 \\
3 & 3.73789 & 83.85195 & 8.58972 \\
4 & -83.93586 & 5.17532 & 4.07039 \\
\hline
\end{tabular}

Fig. 7(a) shows the measuring process in a multi-sensor coordinate measuring machine (Werth Videocheck from Gießen, Germany) and the measured surface. The CMM is equipped with two types of sensors including the WFP (fiber sensor) and the TP200 (trigger probe) which are operated in a clean, thermally controlled, and stable environment. 


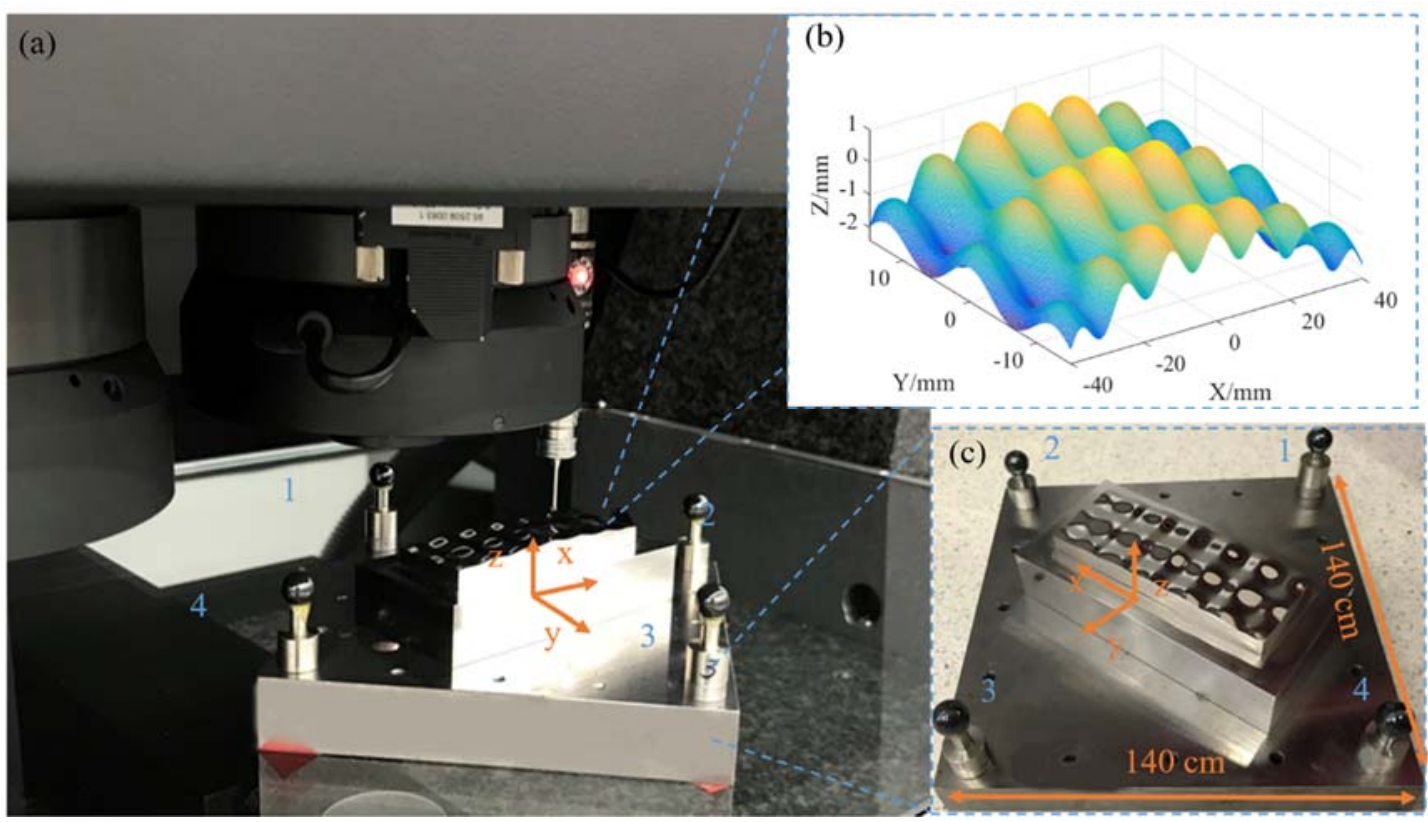

Fig. 7 Measurement of a machined surface (a) measurement process (b) measured surface (c) fiducial aided fixture with the machined workpiece

A total of 2,184 points and 9,063 points were uniformly sampled on the entire surface by the sensor of WFP and the TP200 respectively. In addition, 49 points were sampled on each of the standard balls. Fig. 8 shows examples of the measured fiducials and their position errors as magnified 100 times with different sensors.
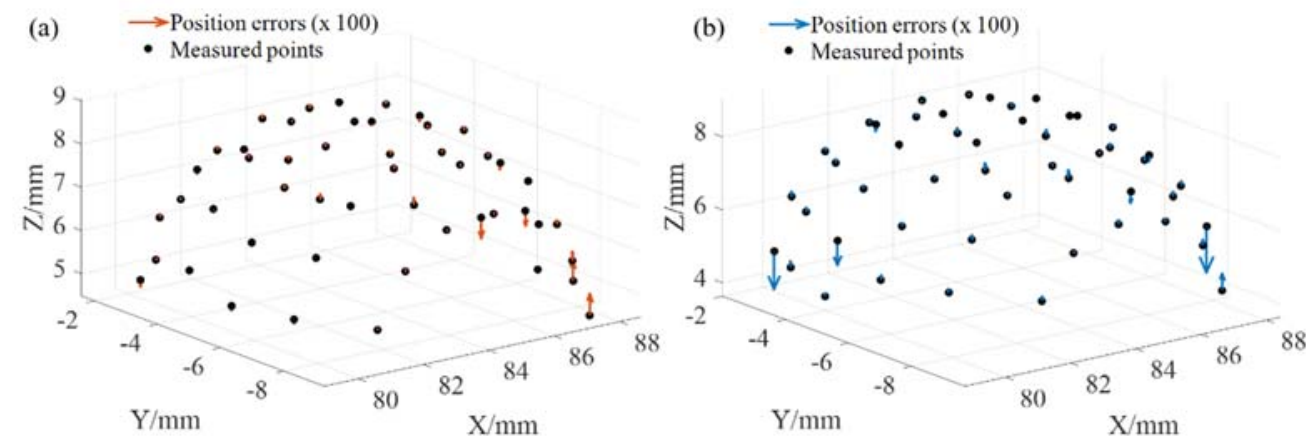

Fig. 8 Measured points and their position errors (error vectors $\times 100$ ) on the fiducials $(a)$ sampled by WFP (b) sampled by TP200

According to Eq. (6), the measuring uncertainty with WFP and TP200 can be easily calculated as:

$$
\begin{aligned}
& \sigma_{\mathrm{WFP}}=2.3 \mu \mathrm{m} \\
& \sigma_{\mathrm{TP} 200}=6.1 \mu \mathrm{m}
\end{aligned}
$$

After the process of fiducial-aided surface registration, the surface residual errors are given in Fig. 9. In order to obtain the information of the fused result, a total of 9,600 $(160 \times 60)$ points were modeled in the grid $x \in[-35,35], y \in[-13,13]$. Both the fused 
results resulting from FADFM and GPBFM are given in Fig. 10. The RMS and PV values of the fused result as well as the dataset sources are listed in Table 3. It is worth noting that the computation time of the two methods was 10 seconds.
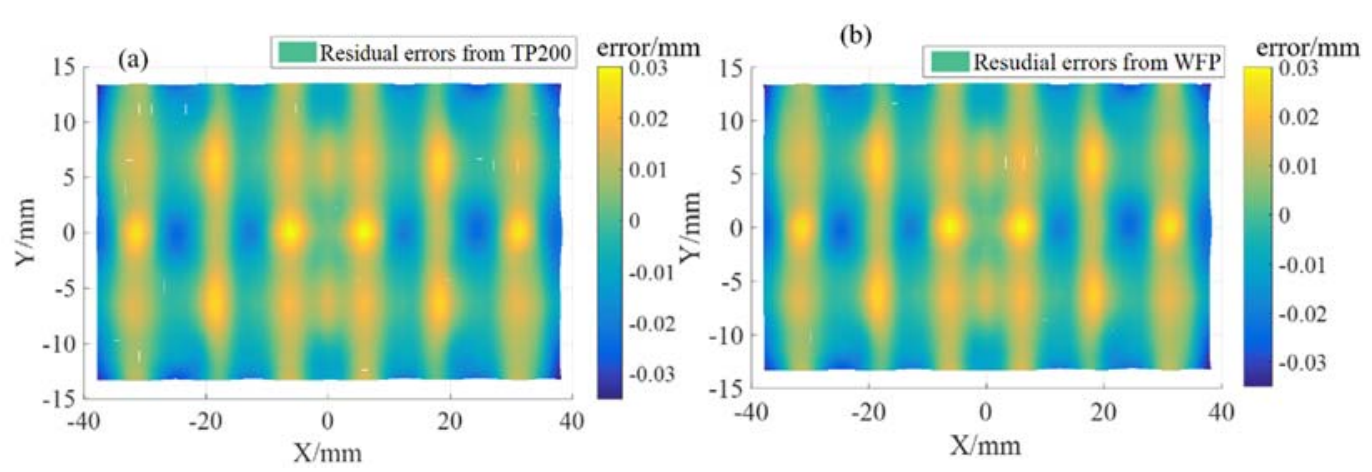

Fig. 9 Residual errors of the machined surface according to (a) TP200 sensor (b) WFP sensor
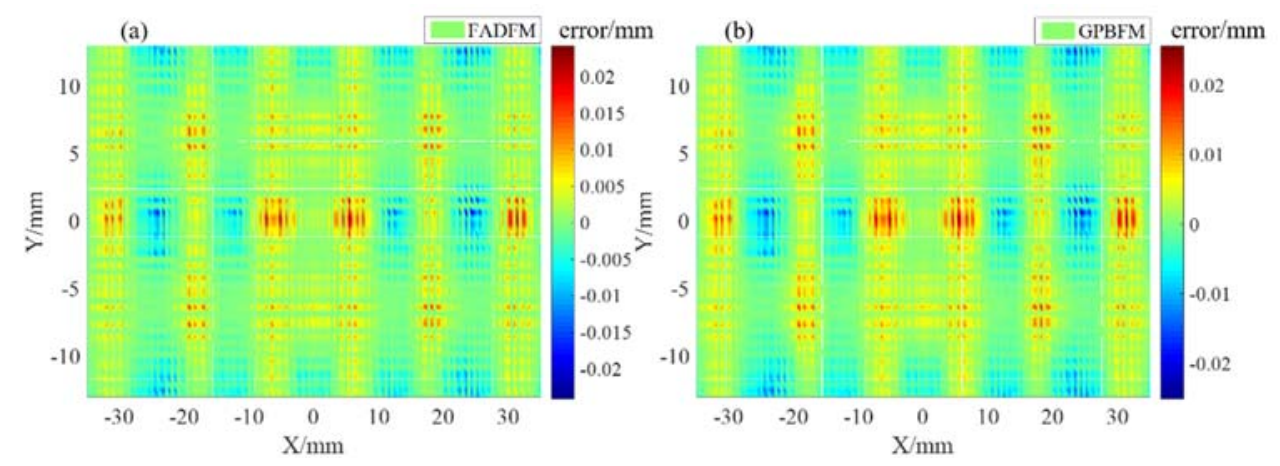

Fig. 10 The fused result of the two methods (a) results of FADFM (b) result of GPBFM

Table 3 Form errors of the machined surface

\begin{tabular}{lllll}
\hline & FADFM & GPBFM & TP200 & WPF \\
\hline Mean of RMS $(\mu \mathrm{m})$ & 3.04 & 4.2 & 11.5 & 10.2 \\
Mean of PV $(\mu \mathrm{m})$ & 32.4 & 50.7 & 65.9 & 64.5 \\
\hline
\end{tabular}

From the table immediately above, it is clear that there is a sharp decrease $(72.5 \%)$ of the measurement uncertainty from each individual sensor up to $11.5 \mu \mathrm{m}$ to the fused result down to $3.04 \mu \mathrm{m}$, which indicates that both of the two methods achieve good results of data fusion. In addition, the proposed FADFM exhibited better performance since there is an improvement of $38.2 \%$ (from $4.2 \mu \mathrm{m}$ to $3.04 \mu \mathrm{m}$ ) for the RMS values and $36.1 \%$ (from $50.7 \mu \mathrm{m}$ to 32.4 ) in terms of the PV values.

On the other hand, the fused results show that the residual errors fluctuate sharply in the micrometer range in the grid of the whole error surface according to Fig. 10 as compared with the relatively smooth error surface as shown in Fig. 9. This is due to the fact that an inadequate number of points were sampled from the sensors and adequate points were sampled in the fused error surface so that the uncertainties in the fusion models were lower. 


\section{Conclusion}

This paper presents a fiducial-aided data fusion method (FADFM) for the measurement of multiscale complex surfaces. A specific fiducial-aided CAD of the designed surface was generated by integrating the designed surface into a fiducialaided fixture with the workpiece mounted on it. To address the key problems in the multi-sensor data fusion, the fiducial aided CAD was employed to carry out two main steps: the fiducial aided surface registration, and a priori information based Gaussian process modelling data fusion. A scatter searching algorithm was employed to solve the nonlinear optimization problem to find the global minimum of the transformation parameters in the transforming positions of the fiducials. Hence, a Gaussian Spectral Mixture kernel was also used to reconstruct the residuals of the datasets and the weighted mean was then determined by the fiducials, which were used to fuse the datasets.

The simulation results show that the uncertainty of the proposed method was reduced up to $3.97 \times 10^{5} \mu \mathrm{m}$ based on the condition that the surface with zero form error. In addition, the computational time of the fusion process was shortened by about 16.7 of the proposed method as compared with the non-prior information based Gaussian process modelling data fusion method. There was still one limitation but it was common in the data fusion in the proposed method. However, this limitation has been improved with the aid of the prior uncertainty of the measured datasets in a weight mean model.

The results of the actual measurement experiments show that the proposed fusion method performed with a sharp decrease $(72.5 \%)$ of the measurement uncertainty as compared with each individual sensor in terms of the RMS value, and there was an improvement of $38.2 \%$ in the RMS values and $36.1 \%$ (from $50.7 \mu \mathrm{m}$ to 32.4 ) in terms of thePV values as compared with the Gaussian process based data fusion method.

The proposed FADFM is able to improve the Gaussian process modelling based data fusion method in terms of reducing measurement uncertainty and computational cost; it also narrows the limitation caused by the large bias of the datasets. Further research should address the limitation by taking into account the systematic error of the datasets.

\section{Acknowledgements}

The work described in this paper was fully supported by a grant from the Research Grants Council of the Government of the Hong Kong Special Administrative Region, China (Project No.: PolyU 15202814). The authors would also like to express their sincere thanks to the Research Committee of The Hong Kong Polytechnic University for their financial support of the project through a PhD studentship (project account code: RUEN).

\section{Reference}

[1] F. Fang, X. Zhang, A. Weckenmann, G. Zhang, and C. Evans, "Manufacturing and measurement of freeform optics," CIRP Annals-Manufacturing Technology, vol. 62, pp. 823-846, 2013. 
[2] W. Lee, S. To, and C. Cheung, "Design and advanced manufacturing technology for freeform optics," ed: The Hong Kong Polytechnic University, 2005.

[3] A. Weckenmann, X. Jiang, K.-D. Sommer, U. Neuschaefer-Rube, J. Seewig, L. Shaw, et al., "Multisensor data fusion in dimensional metrology," CIRP Annals-Manufacturing Technology, vol. 58, pp. 701-721, 2009.

[4] Werth Messtechnik GmbH. WerthVideoCheck UA 400. (2016). Available: http://www.werthmesstechnik.de

[5] G. C. Sharp, S. W. Lee, and D. K. Wehe, "ICP registration using invariant features," IEEE Transactions on Pattern Analysis and Machine Intelligence, vol. 24, pp. 90-102, 2002.

[6] R. Béarée, J.-Y. Dieulot, and P. Rabaté, "An innovative subdivision-ICP registration method for tool-path correction applied to deformed aircraft parts machining," The International Journal of Advanced Manufacturing Technology, vol. 53, pp. 463-471, March 012011.

[7] X. Zhang, L. Jiang, and G. Zhang, "Novel method of positioning optical freeform surfaces based on fringe deflectometry," CIRP Annals-Manufacturing Technology, 2017.

[8] C. Cheung, L. Kong, and M. Ren, "Measurement and characterization of ultra-precision freeform surfaces using an intrinsic surface feature-based method," Measurement Science and Technology, vol. 21, p. 115109, 2010.

[9] M. Zeybek and İ. Şanlıoğlu, "Point cloud filtering on UAV based point cloud," Measurement, vol. 133, pp. 99-111, 2019/02/01/ 2019.

[10] G. M. Krolczyk, R. W. Maruda, P. Nieslony, and M. Wieczorowski, "Surface morphology analysis of Duplex Stainless Steel (DSS) in Clean Production using the Power Spectral Density," Measurement, vol. 94, pp. 464-470, 2016.

[11] S. Wang, C. F. Cheung, M. Ren, and M. Liu, "Fiducial-Aided Robust Positioning of Optical Freeform Surfaces," Micromachines, vol. 9, p. 52, 2018.

[12] V. Mehrad, D. Xue, and P. Gu, "Robust localization to align measured points on the manufactured surface with design surface for freeform surface inspection," ComputerAided Design, vol. 53, pp. 90-103, 8// 2014.

[13] M. Galetto, L. Mastrogiacomo, D. Maisano, and F. Franceschini, "Cooperative fusion of distributed multi-sensor LVM (Large Volume Metrology) systems," CIRP Annals, vol. 64, pp. 483-486, 2015.

[14] A. B. Forbes, "Weighting observations from multi-sensor coordinate measuring systems," Measurement Science and Technology, vol. 23, p. 025004, 2012.

[15] M. Ren, L. Sun, M. Liu, C. Cheung, Y. Yin, and Y. Cao, "A weighted least square based data fusion method for precision measurement of freeform surfaces," Precision Engineering, vol. 48, pp. 144-151, 2017.

[16] J. Wang, L. Pagani, R. Leach, W. Zeng, B. Colosimo, and L. Zhou, Study of weighted fusion methods for the measurement of surface geometry vol. 47, 2017.

[17] B. M. Colosimo, M. Pacella, and N. Senin, "Multisensor data fusion via Gaussian process models for dimensional and geometric verification," Precision Engineering, vol. 40, pp. 199-213, 2015.

[18] M. Liu, C. F. Cheung, C.-H. Cheng, and W. B. Lee, "A gaussian process data modelling and maximum likelihood data fusion method for multi-Sensor CMM measurement of freeform surfaces," Applied Sciences, vol. 6, p. 409, 2016.

[19] M. J. Ren, L. J. Sun, M. Y. Liu, C. F. Cheung, and Y. H. Yin, "A Reconstruction-Registration 
Integrated Data Fusion Method for Measurement of Multiscaled Complex Surfaces," IEEE Transactions on Instrumentation and Measurement, vol. 66, pp. 414-423, 2017.

[20] R. K. Sundaram, A first course in optimization theory: Cambridge university press, 1996.

[21] M. H. Loke and R. Barker, "Rapid least-squares inversion of apparent resistivity pseudosections by a quasi-Newton method," Geophysical prospecting, vol. 44, pp. 131152, 1996.

[22] Z. Ugray, L. Lasdon, J. Plummer, F. Glover, J. Kelly, and R. Martí, "Scatter search and local NLP solvers: A multistart framework for global optimization," INFORMS Journal on Computing, vol. 19, pp. 328-340, 2007.

[23] F. Glover, "A template for scatter search and path relinking," Lecture notes in computer science, vol. 1363, pp. 13-54, 1998.

[24] A. G. Wilson, "Covariance kernels for fast automatic pattern discovery and extrapolation with Gaussian processes," University of Cambridge, 2014.

[25] C. E. Rasmussen. (2010).

Available: http://www.gaussianprocess.org/gpml/code/matlab/doc/ 